\title{
Les médicaments et leur développement pour les enfants
}

\section{Klaus Rose}

Dr med., klausrose Consulting, Pediatric Drug Development \& More, Riehen

\begin{abstract}
La disponibilité croissante de médicaments efficaces et les progrès de la pharmacologie clinique pédiatrique ont entraîné l'exigence de permettre aux enfants de mieux participer aux avancées thérapeutiques pharmaceutiques. Depuis 1997, une législation pédiatrique est en vigueur aux Etats-Unis, celle de l'Union européenne est en vigueur depuis 2007. L'UE tient-elle la promesse qu'elle a faite en 2002 d'une «Amélioration des médicaments pour les enfants»?
\end{abstract}

\section{Résumé}

Les médicaments modernes peuvent sauver des vies, mais ils peuvent aussi s'avérer dangereux. Une évaluation soigneuse du rapport entre les avantages et les risques est fondamentalement nécessaire. C'est avec l'introduction des étiquettes modernes des médicaments qu'a commencé l'indication que le médicament concerné n'a pas été testé ni autorisé pour les enfants. La pédiatrie s'en est longtemps accommodée, mais en 1968, fut formulée la critique que les enfants seraient des "orphelins thérapeutiques». La discussion sur ce sujet a donné naissance à une loi aux USA en 1997, et I'UE a fait de même une décennie plus tard. Depuis 2007, I'UE n'enregistre de nouveaux médicaments qu'avec un plan d'investigation pédiatrique (pediatric investigation plan, PIP). Les promesses de I'UE pour «de meilleurs médicaments pour les enfants" et l'exigence d'une évaluation des médicaments pour les enfants semblent positives à première vue. Mais une analyse critique des décisions du PIP indique un activisme dogmatique des autorités qui ordonnent systématiquement des essais cliniques inutiles et nocifs auprès d'enfants et d'adolescents souffrant de maladies rares et communes. La motivation de cet activisme est complexe, mais des parallèles avec des études douteuses menées durant l'histoire récente sautent inévitablement aux yeux. Les compagnies pharmaceutiques doivent s'engager à réaliser ces études, sans quoi I'EMA n'autorise pas leurs nouveaux médicaments. Le recrutement pour ces études est réalisé dans le monde entier et ne s'arrête pas à la frontière suisse. En Suisse, les médecins, les commissions d'éthique et les parents doivent se montrer très vigilants.

\section{Médicaments, étiquettes et enfants}

La catastrophe de la thalidomide qui a provoqué des milliers de malformations congénitales chez des enfants a montré pour la première fois au monde entier à quel point les médicaments peuvent être dangereux. Depuis 1962, les Etats-Unis exigent une preuve de l'efficacité et de la sécurité des médicaments par le biais d'essais cliniques. Ce fut le début du label moderne (terme anglais désignant l'étiquette). C'est à partir de là qu'il fut souligné que le médicament n'avait pas été testé pour les enfants. Le traitement des enfants était désormais souvent off-label. En 1968, Shirkey a formulé l'idée que les enfants seraient des «orphelins thérapeu- tiques»: ils ne sont pas l'objectif principal du développement des médicaments [1]. Il n'avait pas tout à fait raison: là où il y avait un marché, des médicaments comme les vaccins ou les hormones de croissance ont aussi été développés pour les enfants. Mais souvent, les médicaments n'étaient pas bien adaptés aux enfants ils ne peuvent avaler de comprimés jusqu'à l'âge de 7 ans.

\section{La pharmacologie clinique pédiatrique et la première législation pédiatrique}

Chez les jeunes enfants, l'absorption, la distribution, la métabolisation et l'excrétion (ADME) sont souvent très différentes des adultes, avec un risque de sous-dosage ou de surdosage [2]. Une première loi pédiatrique américaine a offert en 1997 à des sociétés l'extension des brevets pour des études pédiatriques réalisées en accord avec la FDA. Grâce à une loi ultérieure, la FDA put aussi exiger des sociétés pharmaceutiques de réaliser des études pédiatriques.

\section{La législation pédiatrique de l'UE}

Depuis 2007, chaque nouveau médicament nécessite un plan d'investigation pédiatrique (Pediatric Investigation Plan, PIP) approuvé par l'Agence européenne des médicaments (EMA) et son comité pédiatrique (PDCO). Sans PIP, la demande d'autorisation de mise sur le marché pour les adultes n'est pas traitée [3,4]. La législation de l'UE [5] est plus ambitieuse que le modèle américain et est en particulier également valable pour les maladies rares. Aux Etats-Unis, d'intenses discussions ont eu lieu au préalable entre l'industrie, l'académie et les 
autorités de régulation; ici, l'initiative a été prise par la Commission de l'UE et par l'EMA. La première loi américaine n'était pas obligatoire; l'UE a brandi dès le début la menace de non-autorisation pour les adultes.

\section{Le cancer de l'enfant}

Dans les années 1950, les oncologues ont commencé à traiter le cancer de l'enfant avec des cytostatiques pour adultes. C'est ainsi que naquit l'oncologie pédiatrique. La chimiothérapie détruit les cellules malignes, mais non pas de manière sélective. Aujourd'hui, nous sommes au seuil de traitements plus ciblés. Est-ce que les études pédiatriques pour chaque nouveau médicament contre le cancer de l'adulte sont une voie à suivre, ou s'agit-il de la copie mécanique d'une approche qui fonctionnait il y a 50 ans?

\section{La valeur médicale du PIP}

Les résultats du contrôle des études exigées par les PIP sont dévastateurs. Des études réalisées sur des enfants et des adolescents sont exigées pour chaque nouveau médicament, sans le moindre ordre de priorité. Mais les maladies malignes sont rares chez les enfants. L'imatinibe (Gleevec) a été le premier inhibiteur de la tyrosine kinase (TKI) contre la leucémie myéloïde chronique (CML); mais cette dernière est très rare chez les enfants. La CML est fréquente chez les adultes, et il existe des médicaments successeurs pour l'imatinibe (le bosutinibe, le nilotinibe, le ponatinibe). Il n'y a pas assez d'enfants pour des études séparés pour tous les TKI. Mais les sociétés pharmaceutiques doivent s'engager à réaliser de telles études, sans cela l'EMA leur refuse l'autorisation pour les adultes [6]. Il existe maintenant neuf PIPs pour le mélanome, et cinq sociétés cherchent des enfants atteints de mélanome métastatique pour réaliser des essais cliniques. C'est pour de telles études qui ne recruteront jamais assez de patients qu'a été forgée l'expression d'«étude fantôme», et celle d'«otages thérapeutiques» pour leurs malheureux patients suivant le néologisme de Shirkey [7, 8]. Des analyses détaillées ont été publiées sur le mélanome [7], la leucémie [6], la fibrose kystique [9], la sclérose en plaques [10] et les produits allergènes [4].

\section{Le raz-de-marée qui menace}

Toutes les maladies ne sont pas rares chez les enfants, c'est par exemple le cas de la rhinite allergique. Les produits allergènes pour une immunothérapie spécifique (SIT) ont pris un chemin particulier. Les allergènes provenaient tout d'abord de la nature, et avec le temps, ils ont été traités de manière industrielle. La FDA a imposé leur standardisation dans les années 1980. L'UE les a classés comme médicaments en 1989, mais a toléré l'utilisation ultérieure du named patient (patient désigné). En 2008, l'Allemagne a promulgué une loi qui contraint à la ré-homologation des produits SIT [3]. Pour ce faire, il fallait également présenter un PIP dans chaque cas, au total plus de 100. L'Institut Paul Ehrlich (PEI) et l'EMA/ PDCO ont développé ensemble un PIP standard [11-12] pour chaque produit, les mêmes études avec le même design, d'abord avec des adultes, puis avec des enfants et des adolescents, plusieurs centaines par étude et par produit. Après l'étude sur les adultes, les fabricants peuvent déterminer un produit de référence pour les enfants. Cela réduit les études requises d'une durée de 5 ans de 118 à 58 [13]. Une étude SIT actuelle de longue durée prévoit de recruter de 800 à 1000 enfants - c'est une étude. Pour les 57 études restantes, un total de plusieurs dizaines de milliers d'enfants et d'adolescents sera nécessaire. La conclusion des dernières études SIT est exigée pour fin 2031 (!) [4]. Les justifications sont en partie formelles (non conforme aux directives européennes), en partie pseudo-scientifiques: le système immunitaire de l'enfant pourrait mieux fonctionner que celui des adultes; il pourrait y avoir des problèmes de sécurité. Mais pour la sécurité, des registres de traitement seraient suffisants. Les fabricants doivent soit recruter des milliers d'enfants pour des études insensées, soit quitter le marché. Ces études empêchent un traitement efficace dans le groupe placebo, et la progression potentielle vers l'asthme est acceptée froidement. La Suisse a autorisé l'enregistrement ultérieur simplifié des produits allergènes [14]. Personne n'est mort en Suisse parce que des médicaments ne répondaient pas aux exigences de l'UE.

\section{Les fondements spirituels de la législation de l'UE}

Les documents préparés [15] et accompagnant [16] la législation de l'UE exigent l'évaluation clinique des médicaments destinés aux enfants. Mais ils ne font pas de distinction entre ce qui est nécessaire et inutile et ne mentionnent nulle part l'oncologie pédiatrique. Devrions-nous laisser des enfants mourir parce que les médicaments ne sont pas autorisés pour tous les cancers de l'enfance? L'Académie américaine de pédiatrie accepte l'off-label chez les enfants là où cela est sensé et utile [17]. L'UE a quant à elle déformé les préoccupations légitimes qui ont donné naissance à la législation américaine en des exigences dogmatiques d'études cliniques aussi nombreuses que possible à réaliser sur des enfants et des adolescents. 


\section{Atteindre l'âge adulte est une limite juridique et non pas une limite biologique}

Il y a une zone d'incertitude en ce domaine. Au lieu d'un travail constructif, les autorités de l'UE en profitent pour créer un champ d'exploitation pour un activisme enthousiaste.

\section{Les expériences médicales sur les humains}

La réalisation d'expériences médicales sur les humains est complexe et délicate. Une intervention sur le corps dans le cadre d'une expérience peut avoir des conséquences catastrophiques. A l'inverse, un demi-million de personnes ne serait plus en vie en Europe sans l'oncologie pédiatrique. Les expériences criminelles réalisées durant le Troisième Reich ont ébranlé le monde. Et longtemps, personne ne pouvait imaginer que des scientifiques américains pourraient mener des études contraires à l'éthique. Mais en 1966, un courageux clinicien américain publia une liste des études douteuses, toutes publiées dans des revues scientifiques de grande renommée. Comme par exemple un tissu de mélanome transplanté de la fille à la mère, ou l'infection volontaire d'enfants déficients mentaux avec l'hépatite [18]. Létude Tuskegee réalisée en Alabama par l'US Public Health Service (PHS) a elle aussi acquis une bien triste notoriété. Elle a observé de 1932 à 1972 des hommes noirs atteints de syphilis sans les traiter, même lorsque les antibiotiques furent disponibles. Un employé de l'administration qui n'était pas impliqué a tenté d'obtenir l'arrêt de cette étude de manière interne. Sans résultat. Létude n'a pris fin que lorsqu'il s'est adressé à la presse. Des décennies plus tard, le Président Clinton présentait des excuses [19].

\section{Pourquoi?}

Le besoin de réussite personnelle, la soif de pouvoir et la jalousie envers les personnes couronnées de succès sont partout. Il faut un équilibre social pour empêcher les excès. Les études décrites par Beecher ont servi des carrières universitaires - tant que personne ne les observait d'un point de vue critique. L'étude Tuskegee a offert à des bureaucrates des emplois sûrs et du prestige. L'EMA est une construction au-dessus des états de l'UE, elle est officiellement contrôlée par les agences nationales pour l'évaluation des médicaments, la Commission européenne et le Parlement de l'UE, mais elle a une vie propre. Les membres du PDCO proviennent principalement des autorités nationales d'évaluation des médicaments, une minorité sont des médecins cliniques et d'autres profils. C'est avec un haussement d'épaules que la plupart des Européens reconnaissent que des millions de personnes mourraient sans médicaments. Mais les rapports sur la méchante industrie pharmaceutique se vendent bien. Les médicaments devraient être bon marché, développés par de nobles professeurs, ne pas entraîner d'effets secondaires et être écologiques. Les autorités qui font "quelque chose» contre cette méchante industrie et pour les enfants jouissent de la confiance des Européens qui ont foi en l'autorité. La pseudo-science, le sens de la mission, la sécurité de l'emploi et le manque de contrôle de l'EMA se combinent pour créer un sinistre breuvage dont ce seront finalement des enfants et des parents mal orientés qui paieront les pots cassés.

Le tsunami des études pédiatriques insensées de l'UE pourrait se développer en silence. Les compagnies pharmaceutiques ne veulent pas avoir d'ennuis avec les autorités, les cliniciens universitaires ont peu d'intérêt pour les questions d'autorisation des médicaments, et la plupart des pédiatres européens n'ont pas encore perdu toutes leurs illusions sur l'UE. Les protocoles individuels des études pédiatriques PIP semblent à première vue acceptables et sont acceptés par les comités d'éthique. Mais ceux qui ne regardent que les arbres ne voient pas la forêt.

\section{Où allons-nous?}

L'UE menace l'équilibre qui a permis le progrès pharmaceutique. La confiance du public dans les études cliniques réalisées sur des enfants est menacée et la recherche européenne sera isolée sur le plan international. Une première alerte contre les études PIP a été publiée en 2015 [8]. Des organisations de recherche clinique vont également tenter de recruter en Suisse des enfants pour des études insensées. En Suisse, les médecins, les commissions d'éthique et les parents doivent être sur leurs gardes.

\section{Addendum}

Après la remise de l'article, deux études cliniques sur des adolescents atteints de mélanome métastatique ont été abandonnées en raison d'un recrutement trop lent [1, 2]. Cela confirme une nouvelle fois l'actualité de la problématique présentée.

1 BRIM-P: A study of Vemurafenib in pediatric patients with stage IIIC or stage IV melanoma harboring BRAFV600 mutations. https://clinicaltrials.gov/ct2/show/NCTO1519323

2 Phase 2 study of Ipilimumab in children and adolescents $(12$ to $<18$ Years) with previously treated or untreated, unresectable stage III or stage IV malignant melanoma. https://clinicaltrials.gov/ct2/ show/NCT01696045

\section{Disclosure statement}

Après sa formation clinique de spécialiste en médecine générale, l'auteur a travaillé durant vingt années dans la recherche et le développement pour des entreprises pharmaceutiques, dont cinq ans comme global head pediatrics chez Novartis et cinq ans comme global head pediatrics chez Roche. Il est désormais indépendant et conseille des entreprises pharmaceutiques et des institutions académiques sur des questions de développement de médicaments pour les enfants. Une fille de l'auteur est sévèrement handicapée en raison d'une maladie rare. L'auteur ne perçoit ni paiement ni soutien financier pour ses publications dans des revues scientifiques. C'est également le cas pour le présent manuscrit. 


\section{Références}

1 Shirkey H: Therapeutic Orphans. Pediatrics. 1999 Sep;104(3 Pt 2): 583-4. http://pediatrics.aappublications.org/content/pediatrics/104/Supplement_3/583.full.pdf

2 Kearns GL, Abdel-Rahman SM, Alander SW, Blowey DL, Leeder IS, Kauffman RE.: Developmental pharmacology--drug disposition, action, and therapy in infants and children. $\mathrm{N}$ Engl J Med. 2003 Sep 18;349(12):1157-67.

3 Therapieallergene-Verordnung 2008: www.bgbl.de/xaver/bgbl/ start.xav?start=//*\%5B@attr_id=\%27bgbl108s2177.pdf\%27\%5D\# bgbl_\%2F\%2F*\%5B40attr_id\%3D\%27bgbl108s2177. pdf\%27\%5D__1439545547631

4 Rose K \& Kopp MV: Pediatric Investigation Plans (PIPs) for Specific Immunotherapy (SIT): Questionable Contributions To Childhood Health. Pediatr Allergy Immunol.2015 Oct 23

5 VERORDNUNG(EG) Nr. 1901/2006DES EUROPÄISCHEN PARLAMENTS UND DES RATES vom 12. Dezember 2006 über Kinderarzneimittel und zur Änderung der Verordnung (EWG) Nr.1768/92, der Richtlinien 2001/20/EG und 2001/83/EG sowie der Verordnung (EG) Nr.726/2004. Link: http://eur-lex.europa.eu/legal-content/ DE/TXT/?uri=uriserv:OJ.L .2006.378.01.0001.01.

DEU\&toc=OJ:L:2006:378:FULL

6 Rose K \& Walson PD: The contributions of the European Medicines Agency and its pediatric committee to the fight against childhoodleukemia Risk Manag Healthc Policy. 2015 Nov 5;8:185-205. https://www.dovepress.com/the-contributions-of-the-europeanmedicines-agency-and-its-pediatric-c-peer-reviewed-fulltextarticle-RMHP

7 Rose K: European Union Pediatric Legislation Jeopardizes Worldwide, Timely Future Advances in the Care of Children With Cancer. Clinical Therapeutics 2014, 36 (2), 163-177 http://www.clinicaltherapeutics.com/article/SO1492918(14)00018-6/pdf

8 Rose K \& Kummer H: A New Ethical Challenge for Institutional Review Boards (IRBs)/Ethics Committees (ECs) in the Assessment of Pediatric Clinical Trials. Children 2015, 2, 198-210 http://www.mdpi.com/2227-9067/2/2/198

9 Rose K \& Spigarelli MG: Cystic Fibrosis Treatment: A Paradigm for New Pediatric Medicines, Globalization of Drug Development and the Role of the European Medicines Agency. Children 2015, 2(1), 108-130; http://www.mdpi.com/2227-9067/2/1/108
10 Rose K \& Mueller T: Children with Multiple Sclerosis Should Not Become Therapeutic Hostages. Therapeutic Advances in Neurological Disorders 2016 (in press)

11 Englert L, May S, Kaul S \& Vieths S: Die Therapieallergene-Verordnung. Hintergrund und Auswirkungen. Bundesgesundheitsbl 2012 55:351-357 http://www.pei.de/SharedDocs/Downloads/ bundesgesundheitsblatt/2012/2012-therapieallergeneverordnung.pdf? blob=publicationFile\&v $=1$

12 EMA 2015: Allergen Standard PIP: EMA/PDCO Standard Paediatric Investigation Plan for Allergen Products for Specific Immunotherapy Revision 4 http://www.ema.europa.eu/docs/en GB/ document_library/Regulatory_and_procedural_guideline/2009/11/WC500015814.pdf

13 Eichler I \& Sala Soriano E: Close collaboration between academia, industry and drug regulators is required in the development of allergen products for specific immunotherapy in children. Allergy 2011, 66: 999-1004 http://onlinelibrary.wiley.com/doi/10.1111/ j.1398-9995.2011.02582.x/epdf

14 Swissmedic 2014: AW-Verwaltungsverordnung-Vereinfachte Zulassung von Allergenpräparaten. www.swissmedic.ch/ ZL000_00_005d VV

15 European Commission 2002: Better Medicines for Children Proposed regulatory actions on Paediatric medicinal products. http://ec.europa.eu/health/files/pharmacos/docs/doc2002/feb/ cd_pediatrics_en.pdf

16 EMA 2007: The European paediatric initiative: History of the Paediatric Regulation. http://www.ema.europa.eu/docs/en GB/ document_library/Other/2009/09/WC500003693.pdf

17 Fratarelli DA, Galinkin JL, Green TP, Johnson TD, Neville KA Paul IM et al.: Off-label useofdrugsinchildren. Pediatrics. 2014Mar;133(3):563-7 http://pediatrics.aappublications.org/ content/pediatrics/133/3/563.full.pdf

18 Beecher HK. Ethics and clinical research. N Engl J Med 1966; 274 (24): 1354-60

19 Mays VM: The Legacy of the U. S. Public Health Services Study of Untreated Syphilis in African American Men at Tuskegee on the Affordable Care Act and Health Care Reform Fifteen Years After President Clinton's Apology. Ethics Behav. 2012 November 1; 22(6): 411-418. http://www.ncbi.nlm.nih.gov/pmc/articles/PMC3636721/ pdf/nihms458524.pdf 